\title{
SVD-Based Projection for Face Recognition
}

\author{
Chou-Hao Hsu and Chaur-Chin Chen * \\ Department of Computer Science \\ Institute of Information Systems \& Applications \\ National Tsing Hua University \\ Hsinchu 30013, Taiwan \\ E-mail: cchen@cs.nthu.edu.tw \\ Phone: +88635731078 \\ Fax: +88635723694
}

\begin{abstract}
Projection-based face recognition has been widely studied during the past two decades. One of the problems is to require a huge storage space to save the face features obtained from training faces. This paper proposes an SVD-based face retrieval system which requires less memory than the PCA, 2DPCA, Fisher, and 2DFisher approaches. The algorithm tested on the famous ORL (ATETT) face database, consisted of $400112 \times 92$ gray level face images equally contributed by 40 subjects, achieves $97.5 \%$ recognition rate of retrievals.
\end{abstract}

Index Terms-Eigenface, Fisherface, Singular Value Decomposition (SVD).

\section{Introduction}

Projection-based face recognition has been widely studied since the late 1980s. Turk and Pentland [?] investigates the method of Eigenfaces based on (PCA) Principal Component Analysis. Belhumeur et al. [?] investigates the method of Fisherfaces based on Fisher's discriminant analysis. Phillips et al. summarizes a comparison of various algorithms by testing part of FERET face database consisted of 14126 face images contributed by 1199 individuals [?]. Yang et al. [?] investigates the method of 2DPCA by acquiring face features from the right projection of a face image. Li and Yuan [?] investigates 2D-LDA method based on Fisher'r ratio of projections to compute face features. Yang et al. [?] proposes another unsupervised discriminant projection analysis for face feature extraction which takes the local and non-local scatter information into

*This work is supported by Taiwanese National Science Council Grant No. NSC 95-2221-E-007-198. account simultaneously. Dagher and Nachar [?] proposes an algorithm by combining PCA and independent component analysis (ICA) running sequentially. Cho et al. [?] presents an efficient face recognition algorithm based on two directions and 2 dimensional linear discriminant analysis $\left((2 D)^{2} D L D A\right)$ to get the projected face features. Hsu [?] studies various projection methods [?] and proposes a singular value decomposition (SVD) based method for face recognition. The recognition rates reported by the aforementioned research works varied even on testing the commonly used ORL database constructed by AT\&T Laboratories at Cambridge [?] between April 1992 and April 1994 in the lab. We realize that using different subsets of training images and different definitions of recognition rates may obtain various results.

This paper propses a simple algorithm based on singular value decomposition and defines the recognition rate of face image retrievals explicitly. We shall test our algorithm running on ORL database. The remaining of this paper is described as follows. Section 2 introduces an SVD-based algorithm to compute left and right projection vectors, and describes feature extraction by projection and defines the retrieval rate. Section 3 illustrates the ORL face image database, shows the result of performance. Section 4 draws the discussion and conclusion.

\section{SVD-Based Face Represen- tation and Recognition}

Hsu [?] proposed SVD-based face recognition method which applies singular value decomposition for face image reconstruction and recognition. It 
could be viewed as a two-sided 2DPCA method [?]. The matrix inverse of huge image size is also reduced. The face features are stored in the matrix composed of left and right singular vectors of the face image under studies.

Let the gray level face images of $m$ rows and $n$ columns from the jth person out of $K$ persons be denoted as $F_{1}^{(j)}, F_{2}^{(j)}, \cdots, F_{N_{j}}^{(j)} \in R^{m \times n}$ with $1 \leq$ $j \leq K$ and $N_{1}+N_{2}+\cdots+N_{K}=N$, such that $F_{i}^{(j)}(s, t) \in\{0,1, \cdots, 255\}, 1 \leq i \leq N_{j}, 0 \leq s \leq$ $m-1,0 \leq t \leq n-1$. We search for unit vectors $\mathbf{y} \in R^{m}$ and $\mathbf{x} \in R^{n}$ such that $\alpha=\mathbf{y}^{t} \bar{A} \mathbf{x}$ for the average image $\bar{A}$ from the training set to maximize

$$
J(\mathbf{y}, \mathbf{x})=\alpha=\mathbf{y}^{t} \bar{A} \mathbf{x}
$$

The solution from numerical linear algebra supports that the vectors $\mathbf{y}$ and $\mathbf{x}$ are the left and right singular vectors corresponding to the largest singular value.

In practical applications, we first compute the mean image.

$$
\Psi=\frac{1}{N} \sum_{j=1}^{K} \sum_{i=1}^{N_{j}} F_{i}^{(j)}
$$

For each image $A \in R^{m \times n}$, we obtain the left singular vectors $\left\{\mathbf{y}_{i}: 1 \leq i \leq r \leq m\right\}$ and the right singular vectors $\left\{\mathbf{x}_{j}: 1 \leq j \leq c \leq n\right\}$ with the singular values $\sigma_{1} \geq \sigma_{2} \geq \cdots \geq \sigma_{k} \geq 0, k=\min \{m, n\}$.

The face features are stored in the matrix $F \in$ $R^{r \times c}$ acquired by

$$
F=\left[\mathbf{y}_{1}, \mathbf{y}_{2}, \cdots, \mathbf{y}_{\mathbf{r}}\right]^{t} A\left[\mathbf{x}_{1}, \mathbf{x}_{2}, \cdots, \mathbf{x}_{\mathbf{c}}\right]
$$

For reconstruction,

$$
\widehat{A}=\sum_{i=1}^{k} \sigma_{i} \mathbf{y}_{i} \mathbf{x}_{i}^{t}, \quad k \leq \min \{r, c\}
$$

\subsection{SVD-Based Face Representation and Recognition}

Let the gray level face images of $m$ rows and $n$ columns from the jth person out of $K$ persons be denoted as $F_{1}^{(j)}, F_{2}^{(j)}, \cdots, F_{N_{j}}^{(j)} \in R^{m \times n}$ with $1 \leq$ $j \leq K$ and $N_{1}+N_{2}+\cdots+N_{K}=N$, such that $F_{i}^{(j)}(s, t) \in\{0,1, \cdots, 255\}, 1 \leq i \leq N_{j}, 0 \leq s \leq$ $m-1,0 \leq t \leq n-1$. The face image features based on SVD are computed as follows.
(1) Compute the mean face image $\Psi$ by $\Psi=$ $\frac{1}{N} \sum_{j=1}^{K} \sum_{i=1}^{N_{j}} F_{i}^{(j)}$

(2) Apply SVD on $\Psi$ such that $\Psi=U S V^{t}=$ $\sum_{i=1}^{k} \sigma_{i} \mathbf{u}_{i} \mathbf{v}_{i}^{t}$, where $k=\min \{m, n\}$. Denote that $U=\left[\mathbf{u}_{1}, \mathbf{u}_{2}, \cdots, \mathbf{u}_{m}\right], \quad V=$ $\left[\mathbf{v}_{1}, \mathbf{v}_{2}, \cdots, \mathbf{v}_{n}\right], \quad \sigma_{1} \geq \sigma_{2} \geq \cdots \geq \sigma_{k} \geq 0$.

(3) Each training face image $F_{i}^{(j)}$ is transformed into a face feature matrix $X_{i}^{(j)} \in R^{r \times c}$ by $X_{i}^{(j)}=U_{r}^{t} F_{i}^{(j)} V_{c}$

where $r$ and $c$ are user-specified and $U_{r}=$ $\left[\mathbf{u}_{1}, \mathbf{u}_{2}, \cdots, \mathbf{u}_{r}\right], \quad V_{c}=\left[\mathbf{v}_{1}, \mathbf{v}_{2}, \cdots, \mathbf{v}_{c}\right]$.

(4) A test face image $T \in R^{m \times n}$ is transformed into a face feature matrix $Y \in R^{r \times c}$ by $Y=U_{r}^{t} T V_{c}$

(5) Compute the distance between a test face image $T$ and a traning face images $X_{i}^{(j)}$ by $R_{j i}=$ $\delta\left(Y, X_{i}^{(j)}\right)=\left\|Y-X_{i}^{(j)}\right\|_{F}$, a Frobenius norm.

(6) Retrieve the top 8 subjects of the database according to the rank of $R_{j i}$ by

$$
\arg \operatorname{Rank}_{j}\left\{R_{j i}=\delta\left(Y, X_{i}^{(j)}\right), \quad 1 \leq i \leq N_{j}\right\}
$$

\section{Illustration of ORL Face Im- ages and Experiments}

We test our algorithm on the famous ORL (AT\&T) face database which was constructed by AT\&T Laboratories at Cambridge [?]. The ORL has 40 subjects, each contributs 10 face images stored in PGM format with slightly different facial expression, pose, or with/without eyeglasses. We use 200 face images selected from the first 5 images of each subject as training and the remaining face images for testing. Training face images of the subjects $3,8,12,17,1,2,4,5,6,7$ are illustrted in Figure 1.

\subsection{Performance on ORL Database}

In our experiment, we select the parameters $r=12$ and $c=12$ in step (3) based on the ratio of the singular values where the sum of the first 12 singular values achieves approximately $97 \%$ of the sum of all singular values. We not only report the 
rate of recognition but also report the rank of retrievals for better understanding the performance. For each test subject image $T$, we first compute the distance $R(i)$ between this test image and the $i$ th image of the 200 training images according the feature matrices computed from steps (3) and (4) from the SVD-based algorithm as introduced in Section 2. We report the ranks of the best matched subjects according to the distance $R(i)$ from 1 up to 8 . The rate of recognition is shown in Figure 2. The rate indicates the correct recognition occurs when the top $h(1 \leq h \leq 8)$ matches contains at least one successful match. Since there are 5 training images for each subject, if we define a correct recognition for each test image when the best matched rank is within 5 , inclusively. For the aforementioned testing procedures operations, we achieve $97.5 \%$ recognition rate.

The three test images, subject $17-10$, subject 199 , subject 32-7 with none of their corresponding matched subjects is within rank 8 are also interesting. We report these three face images and their corresponding best but wrongly matched face images subject $36-2$, subject $11-4$, subject $15-1$ in Figure 3 for reference.

\section{Discussion and Conclusion}

Face recognition is one of the most popular applications when Biometric Recognition was involved [?]. We propose an SVD-Based face representation and recognition system with very good performance $(97.5 \%)$ on the commonly used ORL (AT\&T) face database. The environments of face image acquisition with experiments to promote the proposed SVD-based face recognition merit further studies.

\section{Acknowledgments}

The authors appreciate the support from Institute of Information Systems and Applications.

\section{References}

[1] P.N. Belhumeur, J.P. Hespanha, and D.J. Kriegman, "Eigenfaces vs. Fisherfaces: Recognition Using Class Specific Linear Projection", IEEE Trans. on Pattern Analysis and Machine Intelligence, vol. 19, no. 7, pp.711-720, 1997.
[2] D.U. Cho, U.D. Chang, K.D. Kim, B.H. Kim, and S.H. Lee, " $(2 D)^{2}$ DLDA for Efficient Face Recognition", First Pacific Rim Symposium, PSIVT 2006, LNCS 4319, pp.314-321, 2006.

[3] I. Dagher and R. Nachar, "Face Recognition Using IPCA-ICA Algorithm", IEEE Trans. on Pattern Analysis and Machine Intelligence, vol. 28, no. 6, pp.996-1000, 2006.

[4] C.H. Hsu, New Projection-Based Features for Face Recognition, M.S. Thesis, National Tsing Hua University, Taiwan, March 2006.

[5] M. Li and B. Yuan, "2D-LDA: A statistical linear discriminant analysis for image matrix", Pattern Recognition Letters, vol. 26, pp.527532, 2005.

[6] P.J. Phillips, H. Moon, S.A. Rizvi, and P.J. Rauss, "The FERET Evaluation Methodology for Face Recognition Face-Recognition Algorithms", IEEE Trans. on Pattern Analysis and Machine Intelligence, vol. 22, no. 10, pp.10901104, 2000.

[7] M.A. Turk and A. Pentland, "Eigenfaces for Recognition", Journal of Cognitive Neuroscience, vol. 3, pp.71-86, 1991.

[8] J. Yang and D. Zhang, A.F. Frangi, and J.Y. Yang, "Two-dimensional PCA: a new approach to appearance-based face representation and recognition", IEEE Trans. on Pattern Analysis and Machine Intelligence, vol. 26, no. 1, pp.131137, 2004.

[9] J. Yang and D. Zhang, Z. Jin, and J.Y. Yang, "Unsupervised Discriminant Projection Analysis for Feature Extraction", Proceedings of International Conference on Pattern Recognition, vol. 1, August 14-16, Hong Kong, China, 2006.

[10] www.face-rec.org

[11] www.cl.cam.ac.uk/Research/DTG/attarchive/facedatabase.htm 


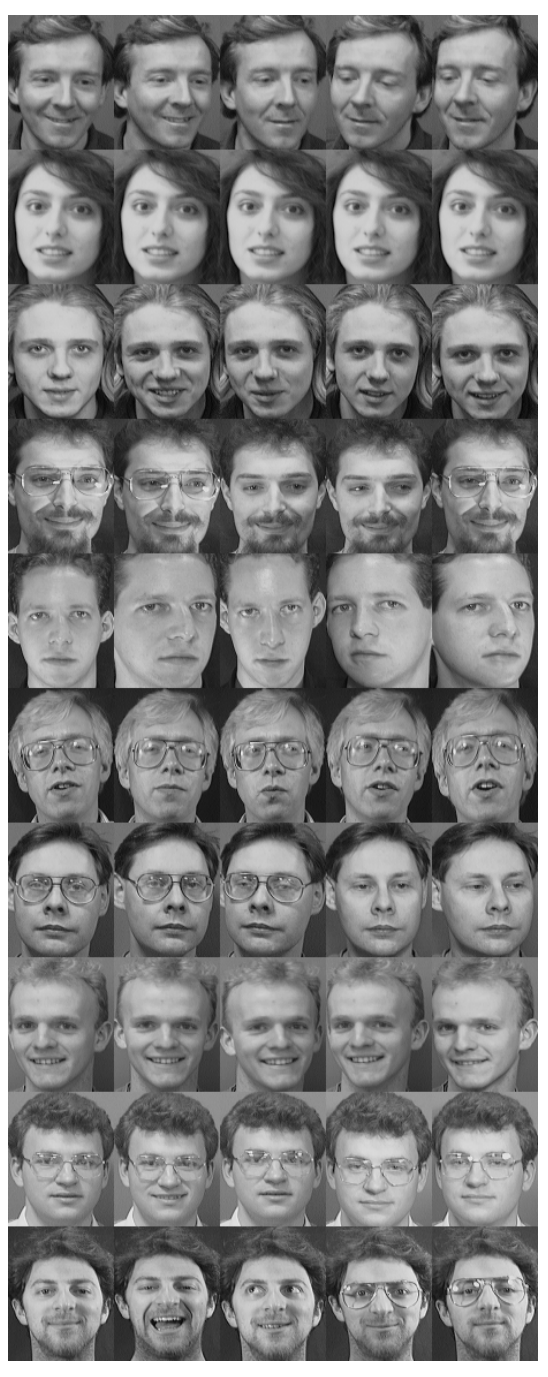

Figure 1: Examples of Face Images of 10 Subjects from ORL Database.

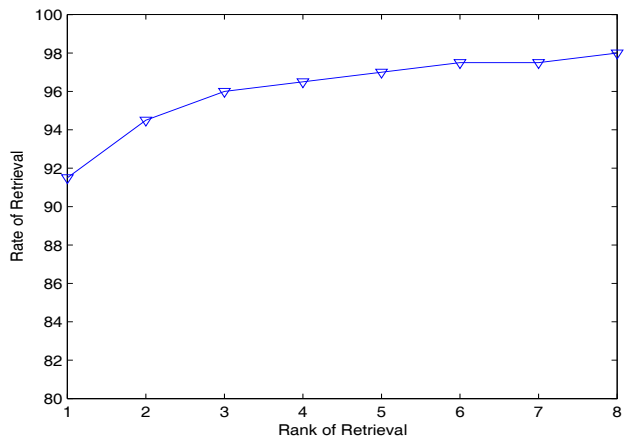

Figure 2: Recognition Rates of Retrievals.

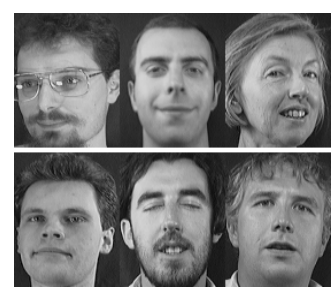

Figure 3: Three Faces and Their Corresponding Best-but-Wrongly Matched Faces. 